\title{
MICROSTRIP CAPACITANCE FOR A CIRCULAR DISK THROUGH MATCHED ASYMPTOTIC EXPANSIONS*
}

\author{
W. C. CHEW $\dagger$ AND J. A. KONG
}

\begin{abstract}
The solution of the potential around two parallel circular disks separated by a dielectric slab is obtained by using the method of matched asymptotic expansions, asymptotic formula for the capacitance has been derived in the limit of small separation $2 \delta$. The formula obtained includes terms of order $\delta$ as well. The mixed boundary value problem is solved by dividing the space around the parallel plates into three regions; the exterior region, the edge region, and the interior region. The solution of the edge region incorporating diegectric effects is obtained by using the Wiener-Hopf technique. The exterior solution of the circular disk problem is obtained by using Hankel transforms. The Hankel transform representation of the exterior solution facilitates the easy derivation of its edge expansion from the Lipschitz-Hankel integrals. The solutions are comiared with Shaw's result for the free-space case [Phys. Fluids, 13 (1970), pp. 1935-1947] and her errors are corrected. Improvement of her approach to the free-space capacitor problem is also discussed.
\end{abstract}

1. Introduction. The problem of the circular disk capacitor in free space has attracted almost a century of attention, mainly due to its mathematical interest [1]-[8]. The advent of microwave integrated circuit has called for a more application-oriented problem consisting of two parallel plates separated by a dielectric slab rather than free space [9]-[11]. Past analysis of the parallel plate capacitor problem in free space has relied on partitioning the space around the disk into a region near the edge and regions away from the edge. Little analytical work has been performed on the microstrip capacitance problem, probably because of the difficulty in obtaining the edge solution, as conformal mapping becomes inapplicable when a dielectric slab instead of free space is involved. Recently, Chew and Kong [11] obtained the solution to the edge region using the Wiener-Hopf technique incorporating the dielectric effect. Consequently, it is possible to use the method of matched asymptotic expansions to obtain analytic solution to the microstrip capacitor to a higher order of accuracy.

The method of matched asymptotic expansions has been widely used in fluid mechanics [12] and recently has been used by Shaw [8] and Cooke [13] to obtain solutions to mixed boundary value problems in homogeneous medium. However, such a method has hardly ever been used to solve mixed boundary value problems occurring in electrostatics, probably because of the added degree of difficulty compounded by the frequent presence of inhomogeneity (e.g., the dielectric layer in the microstrip problem, the layered earth in geophysical probing tools) around the geometry involved. The novelty of matched asymptotic expansions as applied to mixed boundary value problems lies in the reduction of a three-dimensional mixed boundary value problem to a two-dimensional one, or a finite size problem into a semi-infinite one, the solution of which can be more easily obtained. The approach is systematic and, for the circular disk problem, the algebraic manipulations and the integrations involved are complex [14]. However, with the use of MACSYMA, a computer language developed at MIT for symbolic manipulation [15], the task of solving such a problem can be made simpler.

* Received by the editors June 17,1980 , and in revised form January 27,1981 . This work is supported by Schlumberger Doll Research and the Joint Services Electronics Program under contract DAAG-29-78-C0020, the U.S. Energy Research and Development Administration under contract E(11-1)-3070 and the National Aeronautics and Space Administration under grant NSG 1323.

$\dagger$ Formerly at Massachusetts Institute of Technology, presently at Schlumberger-Doll Research, Ridgefield, Connecticut 06877.

$\ddagger$ Department of Electrical Engineering and Computer Science and Research Laboratory of Electronics, Massachusetts Institute of Technology, Cambridge, Massachusetts 02139. 
In this paper, we generalize Shaw's [8] solution of the free-space circular disk capacitor problem to the case when the disks are separated by a dielectric slab. As a modification of her approach, we use the Hankel transform representation for the potential in the exterior region, which enables us to derive the associated surface charged stored and the edge-expansion easily from the integral of Lipschitz-Hankel [16]. Shaw has made use of elliptic integrals for the exterior region solution, and in finding the edge-expansion, she has made use of polynomial approximations. As an improvement, we seek the edge solution using the Wiener-Hopf technique, which has a wider range of applicability than the conformal mapping approach [6]-[8]. Our final formula is compared with Shaw's result when the dielectric is replaced by free space and her errors corrected. However, Shaw should be commended for performing the intricate analysis of the free-space capacitance, as some of her ideas have laid the foundation for our work here.

2. Formulation-the circular coaxial disk problem. The mixed boundary value problem of two circular coaxial disks separated by a dielectric slab of thickness $2 \delta$ is, by the image theorem, the same as the problem of a circular disk separated by a dielectric layer of thickness $\delta$ from a ground plane (Fig. 1). The boundary value problem can be

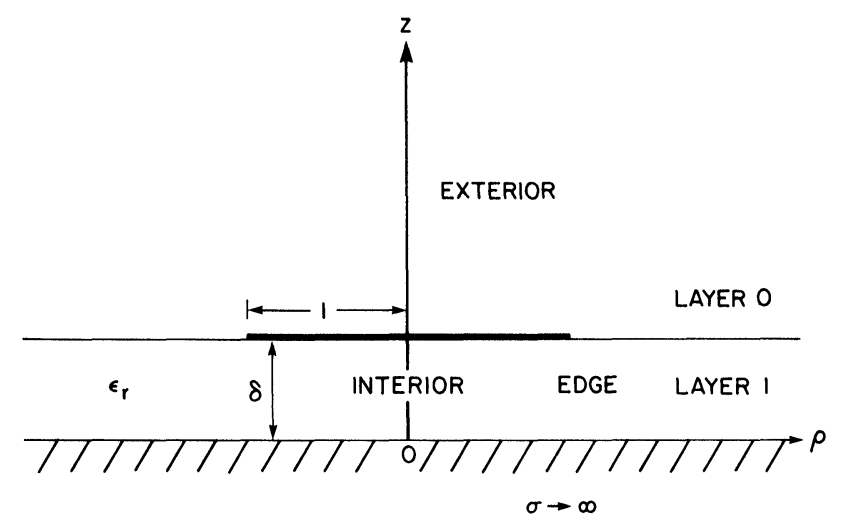

FIG. 1

divided into three regions: the interior region between the parallel plates, the edge region in the vicinity of the edge and the exterior region outside the parallel plates. The potential in each region satisfies the Laplace equation

$$
\frac{1}{\rho} \frac{\partial}{\partial \rho} \rho \frac{\partial}{\partial \rho} V_{i}(\rho, z)+\frac{\partial^{2}}{\partial z^{2}} V_{i}(\rho, z)=0,
$$

where $i$ denotes the layer where the potential is defined, and the boundary conditions are

$$
V_{0}(\rho, \delta)=V_{1}(\rho, \delta) \text { for all } \rho
$$

in particular

$$
\begin{gathered}
V_{0}(\rho, \delta)=V_{1}(\rho, \delta)=1 \text { for } \rho<1, \\
V_{1}(\rho, 0)=0 \text { for all } \rho \\
\frac{\partial V_{0}}{\partial z}(\rho, \delta)=\varepsilon_{r} \frac{\partial V_{1}}{\partial z}(\rho, \delta) \text { for } \rho>1
\end{gathered}
$$


At this juncture, it is appropriate to introduce stretched coordinate systems for the edge and interior regions to emphasize the singularity of the edge region and the $z$-variation of the interior region. The transformation for the edge region is given by

$$
X=\frac{\rho-1}{\delta}, \quad Y=\frac{z}{\delta} .
$$

The transformation for the interior region is given by

$$
Y=\frac{z}{\delta}
$$

while the $\rho$-coordinate is not magnified. Consequently, the equation written in terms of edge variables is

$$
\left(\frac{\partial^{2}}{\partial X^{2}}+\frac{\delta}{1+\delta X} \frac{\partial}{\partial X}+\frac{\partial^{2}}{\partial Y^{2}}\right) \Phi_{i}(X, Y)=0
$$

and the boundary condition is

$$
\Phi_{0}(X, 1)=\Phi_{1}(X, 1) \text { for all } X
$$

in particular

$$
\begin{gathered}
\Phi_{0}(X, 1)=\Phi_{1}(X, 1)=1 \text { for } X<0, \\
\Phi_{1}(X, 0)=0 \text { for all } X \\
\frac{\partial \Phi_{0}}{\partial Y}(X, 1)=\varepsilon_{r} \frac{\partial \Phi_{1}}{\partial Y}(X, 1) \text { for } X>0 .
\end{gathered}
$$

The equation in terms of the interior variables is

$$
\left(\frac{\delta^{2} \partial}{\rho \partial \rho} \rho \frac{\partial}{\partial \rho}+\frac{\partial^{2}}{\partial Y^{2}}\right) \Psi_{i}(\rho, Y)=0
$$

and the boundary conditions are transformed accordingly. In the following analysis, we shall use $V_{0}(\rho, z), \Phi_{i}(X, Y)$ and $\psi_{1}(\rho, Y)$ consistently to denote the exterior solution, the edge solution and the interior solution respectively.

\section{Method of solution.}

3.1. The first approximation. In the limit when $\delta \rightarrow 0,(1),(5)$ and (7), with their respective boundary conditions, give rise to solutions emphasizing their respective regions. For example, in the limit when $\delta \rightarrow 0$, (1), with its boundary conditions, gives the exterior-limit solution

$$
V_{0}^{(0)}(\rho, z)=\int_{0}^{\infty} J_{1}(\lambda) J_{0}(\lambda \rho) e^{-\lambda z} d \lambda,
$$

which can be shown easily to satisfy the boundary condition

$$
\begin{array}{ll}
V_{0}^{(0)}(\rho, 0)=0, & \rho>1, \\
V_{0}^{(0)}(\rho, 0)=1, & \rho<1 .
\end{array}
$$

The edge-limit solution when $\delta \rightarrow 0$ is solvable by the Wiener-Hopf technique [11], which is described briefly in Appendix A. The solution is

$$
\Phi_{0}^{(0)}(X, Y)=\int_{-\infty}^{\infty} \phi_{+}(\lambda) \frac{G_{-}(\lambda)}{G_{-}(0)} e^{i \lambda X-\alpha(Y-1)} d \lambda
$$




$$
\Phi_{1}^{(0)}(X, Y)=\int_{-\infty}^{\infty} \phi_{+}(\lambda) \frac{G_{-}(\lambda)}{G_{-}(0)}\left[\frac{e^{\alpha(Y-1)}-e^{-2 \alpha-\alpha(Y-1)}}{1-e^{-2 \alpha}}\right] e^{i \lambda X} d \lambda,
$$

where the functional variables are defined in Appendix A. The interior-limit solution is given by

$$
\psi_{1}^{(0)}(\rho, Y)=Y
$$

in the interior region.

In the matched asymptotic expansion, we adopt the matching principle [12, p. 220] given by

the edge expansion to order $\Delta(\delta)$ of $\left(\right.$ the $\left\{\begin{array}{l}\text { exterior } \\ \text { interior }\end{array}\right\}$ expansion to order $\left.\beta(\delta)\right)$

$=$ the $\left\{\begin{array}{l}\text { exterior } \\ \text { interior }\end{array}\right\}$ expansion to order $\beta(\delta)$ of (the edge expansion to order $\left.\Delta(\delta)\right)$,

where the comparison is done in a common variable. Using the approximation derived by Eason et al. [16] for the edge-expansion of (8) and the asymptotic approximation derived in Appendix A for the exterior expansion of (10) and the interior expansion (11), we can show that the leading order approximations in each region satisfy the matching principle.

3.2. The higher-order approximation. The exterior expansion of the edge solution is given in terms of exterior variables by (see Appendix A, (A.12))

$$
\begin{gathered}
\Phi_{0}^{(0)}\left(\frac{\rho-1}{\delta}, \frac{z}{\delta}\right) \sim \frac{1}{\pi} \tan ^{-1} \frac{z}{\rho-1}-\frac{\delta \ln \delta}{\pi^{2} \varepsilon_{r}} \frac{z}{(\rho-1)^{2}+z^{2}} \\
-\frac{\delta}{\pi^{2} \varepsilon_{r}}\left\{\frac{\rho-1}{(\rho-1)^{2}+z^{2}} \tan ^{-1} \frac{z}{(\rho-1)}\right. \\
-\frac{z}{(\rho-1)^{2}+z^{2}}\left[\ln \left[(\rho-1)^{2}+z^{2}\right]^{1 / 2}+A\right] \\
\left.+\frac{\pi(\rho-1)}{(\rho-1)^{2}+z^{2}}\left(\varepsilon_{r}-1\right)\right\}, \\
\quad \delta \rightarrow 0, \quad \rho, z \text { fixed, }
\end{gathered}
$$

where $A$ is a constant defined by

$$
A=-2 \varepsilon_{r} \sum_{n=1}^{\infty}\left(\frac{1-\varepsilon_{r}}{1+\varepsilon_{r}}\right)^{n} \ln (n)+\varepsilon_{r} \ln \pi+\left(\varepsilon_{r}-1\right) \ln 2+1 .
$$

This leads to the perturbation series for the exterior solution as

$$
V_{0}(\rho, z) \sim V_{0}^{(0)}(\rho, z)+\delta \ln \delta V_{0}^{(1)}(\rho, z)+\delta V_{0}^{(2)}(\rho, z), \quad \delta \rightarrow 0
$$

When the above is substituted into (1) and the boundary conditions expanded around $\delta=0$, we obtain the following equations and boundary conditions to be satisfied by the higher order terms of $V_{0}(\rho, z)$,

$$
\left(\frac{1}{\rho} \frac{\partial}{\partial \rho} \rho \frac{\partial}{\partial \rho}+\frac{\partial^{2}}{\partial z^{2}}\right) V_{0}^{(1)}(\rho, z)=0,
$$


with the boundary condition

$$
V_{0}^{(1)}(\rho, 0)=0,
$$

and satisfying the matching condition provided by (14a). Also,

$$
\left(\frac{1}{\rho} \frac{\partial}{\partial \rho} \rho \frac{\partial}{\partial \rho}+\frac{\partial^{2}}{\partial z^{2}}\right) V_{0}^{(2)}(\rho, z)=0,
$$

with the boundary conditions

$$
\begin{aligned}
& V_{0}^{(2)}(\rho, 0)=-\frac{\varepsilon_{r}-1}{\varepsilon_{r}} \frac{\partial V_{0}^{(0)}}{\partial z}(\rho, 0), \quad \rho>1, \\
& V_{0}^{(2)}(\rho, 0)=-\frac{\partial V_{0}^{(0)}}{\partial z}(\rho, 0), \quad \rho<1,
\end{aligned}
$$

and the matching condition provided by (14a). The solution to (16) is equivalent to an eigensolution corresponding to a singular Dirac delta function of dipole density at the ring $\rho=1$ given by

$$
D(\rho)=-\frac{2 \varepsilon_{0} \delta(\rho-1)}{\pi \varepsilon_{r} \rho} .
$$

In Hankel transform representation, it is given by

$$
V_{0}^{(1)}(\rho, z)=-\frac{1}{\pi \varepsilon_{r}} \int_{0}^{\infty} \lambda J_{0}(\lambda) J_{0}(\lambda \rho) e^{-\lambda z} d \lambda .
$$

It can be readily verified that (21) satisfies the matching condition provided by (14a) by using the approximation of Eason et al. [16] to find the edge expansion of (21).

The solution of (18) is slightly more involved. From (8), we find

$$
\frac{\partial V_{0}^{(0)}}{\partial z}(\rho, 0)=-\int_{0}^{\infty} \lambda J_{1}(\lambda) J_{0}(\lambda \rho) d \lambda=-\frac{2 E(\rho)}{\pi\left(1-\rho^{2}\right)},
$$

where $E(\rho)$ is an elliptic integral. As such, we derive that

$$
\begin{aligned}
V_{0}^{(2)}(\rho, z)= & \frac{2}{\pi \varepsilon_{r}} \int_{0}^{\infty} d \lambda J_{0}(\lambda \rho) e^{-\lambda z} \int_{0}^{1} \frac{d b}{1-b}\left[\frac{b E(b)}{1+b} J_{0}(\lambda b)-\frac{J_{0}(\lambda)}{2}\right] \\
& +\frac{\left(\varepsilon_{r}-1\right)}{\varepsilon_{r}} \int_{0}^{\infty} \lambda J_{1}(\lambda) J_{0}(\lambda \rho) e^{-\lambda z} d \lambda+\frac{A}{\pi \varepsilon_{r}} \int_{0}^{\infty} \lambda J_{0}(\lambda) J_{0}(\lambda \rho) e^{-\lambda z} d \lambda .
\end{aligned}
$$

In the above, the last term is an eigensolution added to satisfy the matching condition provided by $(14 a)$.

The interior expansion of the edge solution is given by (see Appendix A)

$$
\Phi_{1}^{(0)}(X, Y) \sim Y+\text { exponentially small terms, } \quad X \rightarrow-\infty,
$$

implying that higher order terms of the interior solution is transcendentally small. 
To obtain the form of the perturbation series for the edge solution, we find the edge expansion of the exterior solution which is verified in Appendix B as

$$
\begin{aligned}
& V_{0}(1+\delta X, \delta Y) \sim \frac{1}{\pi} \tan ^{-1}(Y / X)+\frac{1}{\pi^{2} \varepsilon_{r}\left(X^{2}+Y^{2}\right)}\left\{Y\left[\ln \left(X^{2}+Y^{2}\right)^{1 / 2}+A\right]\right. \\
&\left.-X \tan ^{-1}(Y / X)-\pi\left(\varepsilon_{r}-1\right) X\right\} \\
&+\frac{\delta}{2 \pi} \ln \left[\delta \frac{\left(X^{2}+Y^{2}\right)^{1 / 2}}{8}\right]\left[Y-\frac{1}{\pi \varepsilon_{r}} \tan ^{-1}(Y / X)-1+\frac{1}{\varepsilon_{r}}\right] \\
&-\frac{\delta Y}{2 \pi^{2} \varepsilon_{r}\left(X^{2}+Y^{2}\right)}\left\{X\left[\ln \left(X^{2}+Y^{2}\right)^{1 / 2}+A\right]\right. \\
&\left.+Y \tan ^{-1}(Y / X)+\pi\left(\varepsilon_{r}-1\right) Y\right\}, \quad \delta \rightarrow 0 .
\end{aligned}
$$

This indicates that the perturbation series for the edge solution is of the form

$$
\Phi_{i}(X, Y) \sim \Phi_{i}^{(0)}(X, Y)+\delta \ln \delta \Phi_{i}^{(1)}(X, Y)+\delta \Phi_{i}^{(2)}(X, Y), \quad \delta \rightarrow 0 .
$$

When the above is substituted into (5), the equation to be satisfied by $\Phi_{i}^{(1)}(X, Y)$ becomes

$$
\left(\frac{\partial^{2}}{\partial X^{2}}+\frac{\partial^{2}}{\partial Y^{2}}\right) \Phi_{i}^{(1)}(X, Y)=0
$$

The boundary conditions are

$$
\Phi_{0}^{(1)}(X, 1)=\Phi_{1}^{(1)}(X, 1) \text { for all } X,
$$

in particular

$$
\begin{gathered}
\Phi_{0}^{(1)}(X, 1)=\Phi_{1}^{(1)}(X, 1)=0 \text { for } X<0, \\
\Phi_{1}^{(1)}(X, 0)=0 \text { for all } X, \\
\frac{\partial \Phi_{0}^{(1)}}{\partial Y}(X, 1)=\varepsilon_{r} \frac{\partial \Phi_{1}^{(1)}}{\partial Y}(X, 1) \text { for } X>0,
\end{gathered}
$$

and the matching condition is provided by (25). The equation satisfied by $\Phi_{i}^{(2)}(X, Y)$ is

$$
\left(\frac{\partial^{2}}{\partial X^{2}}+\frac{\partial^{2}}{\partial Y^{2}}\right) \Phi_{i}^{(2)}(X, Y)=-\frac{\partial \Phi_{i}^{(0)}(X, Y)}{\partial X}
$$

and the boundary condition is the same as that of $\Phi_{i}^{(1)}(X, Y)$. The matching condition is supplied by (25).

By inspection, the solution to (27) and (28) is

$$
\begin{aligned}
& \Phi_{0}^{(1)}(X, Y)=-\frac{1}{2 \pi \varepsilon_{r}} \Phi_{0}^{(0)}(X, Y)-\frac{1}{2 \pi}\left(1-\frac{1}{\varepsilon_{r}}-Y\right), \\
& \Phi_{1}^{(1)}(X, Y)=-\frac{1}{2 \pi \varepsilon_{r}} \Phi_{1}^{(0)}(X, Y)+\frac{Y}{2 \pi \varepsilon_{r}} .
\end{aligned}
$$

The particular solution to (29) is given by

$$
\Phi_{i p}^{(2)}(X, Y)=-\frac{X}{2} \Phi_{i}^{(0)}(X, Y) .
$$


In order to satisfy the homogeneous boundary condition at $Y=1, X<0$, a homogeneous solution to (29) is necessary. Since

$$
\int_{0}^{X} \Phi_{i}^{(0)}\left(X^{\prime}, Y\right) d X^{\prime}
$$

is also a harmonic function, a homogeneous solution can be obtained by integrating (10) and (11). Consequently, a homogeneous solution, which when added to the particular solution causes the homogeneous boundary condition and the matching condition to be satisfied, is

$$
\begin{aligned}
\Phi_{o h}^{(2)}(X, Y)= & \frac{1}{2} \int_{-\infty}^{\infty} \frac{\phi_{+}(\lambda) G_{-}(\lambda)}{i \lambda G_{-}(0)}\left[e^{i \lambda X-\alpha(Y-1)}-\varepsilon_{r}(Y-1)-1\right] d \lambda \\
& -[A+\ln 8-1] \Phi_{0}^{(1)}(X, Y), \\
\Phi_{1 h}^{(2)}(X, Y)= & \frac{1}{2} \int_{-\infty}^{\infty} \frac{\phi_{+}(\lambda) G_{-}(\lambda)}{i \lambda G_{-}(0)}\left(\frac{e^{\alpha(Y-1)}-e^{-2 \alpha-\alpha(Y-1)}}{1-e^{-2 \alpha}} e^{i \lambda X}-Y\right) d \lambda \\
- & {[A+\ln 8-1] \Phi_{1}^{(1)}(X, Y) . }
\end{aligned}
$$

The satisfaction of the matching condition by $\Phi_{i}^{(2)}=\Phi_{i p}^{(2)}+\Phi_{i h}^{(2)}$ is proven in Appendix A by finding the asymptotic approximations for the above expressions.

4. The capacitance. The capacitance is obtained from the total charge $Q$ on the disk divided by the potential difference. For the configuration of Fig. 1, it is simply $Q$.

The charge contained in the exterior surface of the disk within a circle of radius $\rho$ is given by

$$
q_{0}(\rho)=-2 \pi \varepsilon_{0} \int_{0}^{\rho} \rho^{\prime} \frac{\partial V_{0}\left(\rho^{\prime}, 0^{+}\right)}{\partial z} d \rho^{\prime} \sim q_{0}^{(0)}(\rho)+\delta \ln \delta q_{0}^{(1)}(\rho)+\delta q_{0}^{(2)}(\rho), \quad \rho<1,
$$

where

$$
\begin{gathered}
q_{0}^{(0)}(\rho)=2 \pi \varepsilon_{0} \rho \int_{0}^{\infty} J_{1}(\lambda) J_{1}(\lambda \rho) d \lambda \\
q_{0}^{(1)}(\rho)=-2 \frac{\varepsilon_{0} \rho}{\varepsilon_{r}} \int_{0}^{\infty} \lambda J_{0}(\lambda) J_{1}(\lambda \rho) d \lambda \\
q_{0}^{(2)}(\rho)=\lim _{z \rightarrow 0+}\left\{\frac{4 \varepsilon_{0} \rho}{\varepsilon_{r}} \int_{0}^{\infty} d \lambda J_{1}(\lambda \rho) e^{-\lambda z} \int_{0}^{1} \frac{d b}{1-b}\left[\frac{b E(b)}{1+b} J_{0}(\lambda b)-\frac{J_{0}(\lambda)}{2}\right]\right\} \\
+\frac{2 A \varepsilon_{0} \rho}{\varepsilon_{r}} \int_{0}^{\infty} \lambda J_{0}(\lambda) J_{1}(\lambda \rho) d \lambda .
\end{gathered}
$$

The charge stored in the interior surface of the disk is given by

$$
q_{1}(\rho) \sim \frac{\pi \varepsilon_{1} \rho^{2}}{\delta}, \quad \rho<1
$$

The charge stored in the edge region in an annulus where $1-\delta W<\rho<1$ is

$$
\begin{aligned}
Q(-W) & =-2 \pi \varepsilon_{0} \int_{-W}^{0}\left[\frac{\partial \Phi_{0}(X, 1)}{\partial Y}-\varepsilon_{r} \frac{\partial \Phi_{1}(X, 1)}{\partial Y}\right](1+\delta X) d X \\
& \sim Q^{(0)}(-W)+\delta \ln \delta Q^{(1)}(-W)+\delta Q^{(2)}(-W),
\end{aligned}
$$


where

$$
\begin{gathered}
Q^{(0)}(-W)=2 \pi \int_{-W}^{0} \Sigma^{(0)}(X) d X, \\
Q^{(1)}(-W)=-\frac{1}{\varepsilon_{r}} \int_{-W}^{0} \Sigma^{(0)}(X) d X \\
Q^{(2)}(-W)=-\pi W \int_{-W}^{0} \Sigma^{(0)}(X) d X+\frac{[A+\ln 8-1]}{\varepsilon_{r}} \int_{-W}^{0} \Sigma^{(0)}(X) d X, \\
\Sigma^{(0)}(X)=-\varepsilon_{0}\left[\frac{\partial \Phi_{0}^{(0)}(X, 1)}{\partial Y}-\varepsilon_{r} \frac{\partial \Phi_{1}^{(0)}(X, 1)}{\partial Y}\right] .
\end{gathered}
$$

The total charge on the disk and thus the capacitance $C$ is given by

$$
C=q_{0}(1-\delta W)+q_{1}(1-\delta W)+Q(-W) .
$$

The edge expansion of $q_{0}(1-\delta W)$ and the outer expansion of $Q(-W)$ are derived in Appendix B. Hence, for fixed $W$,

$$
\begin{aligned}
& q_{0}(1-\delta W)+q_{1}(1-\delta W) \\
& \sim \frac{\pi \varepsilon_{1}}{\delta}-2 \pi \varepsilon_{1} W-2 \varepsilon_{0} \ln W-\frac{2 \varepsilon_{0}}{\pi \varepsilon_{r} W}(\ln W+A)+2 \varepsilon_{0}\left[\ln \frac{8}{\delta}-2\right]+\delta \ln ^{2} \delta \frac{\varepsilon_{0}}{\pi \varepsilon_{r}} \\
& +\delta \ln (\delta) \varepsilon_{0}\left\{W+\frac{1}{\pi \varepsilon_{r}}(\ln W-A-2 \ln 8+2)\right\} \\
& +\delta\left\{\varepsilon_{0} W\left(\ln \frac{W}{8}+1\right)-\frac{\varepsilon_{0}}{\pi \varepsilon_{r}}(A+\ln 8-2) \ln W\right. \\
& \left.\quad+\frac{\varepsilon_{0} A \ln 8}{\pi \varepsilon_{r}}+\pi \varepsilon_{1} W^{2}+\frac{\varepsilon_{0}}{\pi \varepsilon_{r}}\left[\left(\ln \frac{1}{8}+1\right)^{2}-2\right]\right\}, \quad \delta \rightarrow 0,
\end{aligned}
$$

$Q(-W)=\left\{2 \pi-\frac{\delta \ln \delta}{\varepsilon_{r}}+\frac{\delta[A+\ln 8-1]}{\varepsilon_{r}}-\delta \pi W\right\} \int_{-W}^{0} \Sigma^{(0)}(X) d X$

(40)

$$
\sim 2 \pi \varepsilon_{1} W+2 \varepsilon_{0} \ln W+2 \varepsilon_{0} A+\frac{2 \varepsilon_{0}}{\pi \varepsilon_{r} W}(\ln W+A)
$$

$$
\begin{aligned}
& -\delta \ln \delta \varepsilon_{0}\left\{W+\frac{1}{\pi \varepsilon_{r}} \ln W+\frac{A}{\pi \varepsilon_{r}}+\cdots\right\} \\
& +\delta\left\{-\pi \varepsilon_{1} W^{2}-\varepsilon_{0} W\left[\ln \frac{W}{8}+1\right]\right. \\
& \left.+\frac{\varepsilon_{0}}{\pi \varepsilon_{r}}[A+\ln 8-2] \ln W+\frac{\varepsilon_{0}}{\pi \varepsilon_{r}} A[A+\ln 8-2]\right\}, \quad W \rightarrow \infty .
\end{aligned}
$$

Consequently, the capacitance is given by

$$
C \sim \frac{\pi \varepsilon_{1}}{\delta}+2 \varepsilon_{0}\left[\ln \frac{8}{\delta}-2+A\right]+\frac{\delta \varepsilon_{0}}{\pi \varepsilon_{r}}\left[\left(\ln \frac{8}{\delta}+A-1\right)^{2}-2\right]
$$


When $\varepsilon_{r}=1, A=\ln \pi+1$ and

$$
C \sim \frac{\pi \varepsilon_{0}}{\delta}+2 \varepsilon_{0}\left[\ln \frac{8 \pi}{\delta}-1\right]+\frac{\delta \varepsilon_{0}}{\pi}\left[\ln ^{2} \frac{\delta}{8 \pi}-2\right]
$$

The above contains an extra term of $-2 \delta \varepsilon_{0} / \pi$ when compared with Shaw's result. This is because the constant term of -1 in the integral approximation (B.6a) is left out [14, p. 72].

6. Conclusion. We have derived the matched asymptotic expansions of the potential around the circular microstrip disk as well as its capacitance. The formula for the capacitance assumes simple forms and can be easily computed. Even though the analysis was performed for small $\delta$, the formula is found to give an error of no greater than $8 \%$ for $\delta \leqq 1$ when compared with numerically obtained results [10], which is typical of perturbation analysis. It thus seems that the method of matched asymptotic expansions is a viable approach for analytic solutions of microstrip problems, which has wide ranging applications [9]-[10]. The solution for the microstrip problem is possible due to the availability of the edge solution using the Wiener-Hopf technique.

Appendix A. The two-dimensional problem of the edge solution can be solved by the Wiener-Hopf technique indirectly. First we find the solution of the Helmholtz wave equation $\left(\nabla^{2}+k^{2}\right) \Phi_{i}^{(0)}(X, Y)=0$ (where $k$ is a complex number) which satisfies the boundary conditions provided by (6). We retrieve the solution to Laplace's equation by letting $k \rightarrow 0$. The problem can be formulated as dual integral equations given by

$$
\begin{gathered}
\Phi_{0}^{(0)}(X, Y=1)=\int_{-\infty}^{\infty} \sigma(\lambda) g(\lambda) e^{i \lambda x} d \lambda=1, \quad X<0, \\
\Sigma^{(0)}(X)=\int_{-\infty}^{\infty} \sigma(\lambda) e^{i \lambda x} d \lambda=0, \quad X>0,
\end{gathered}
$$

where

$$
g(\lambda)=\frac{2}{\varepsilon_{0}+\varepsilon_{1}}\left(\frac{\sinh \alpha}{\alpha}\right)\left(\frac{e^{-\alpha}}{1-r_{01} e^{-2 \alpha}}\right),
$$

$$
\begin{gathered}
\alpha=\sqrt{\lambda^{2}-k^{2}}, \\
r_{01}=\frac{1-\varepsilon_{r}}{1+\varepsilon_{r}} .
\end{gathered}
$$

In (A.2b), we choose the branch of square root so that $\operatorname{Re}[\alpha]>0$ in order for the wave to satisfy the radiation condition. The above can be solved by the standard WienerHopf technique [17] provided that $g(\lambda)$ can be factorized as

$$
g(\lambda)=\frac{2}{\varepsilon_{0}+\varepsilon_{1}} G_{+}(\lambda) G_{-}(\lambda)
$$

where $G_{+}(\lambda)$ and $G_{-}(\lambda)$ are analytic functions in the upper and lower half-plane, respectively, sharing a common strip of analyticity. $g(\lambda)$ can be factorized by expanding out its denominator, and applying Cauchy's integral theorem [17]. As such,

$$
G_{ \pm}(\lambda)=L_{ \pm}(\lambda) e^{-F_{ \pm}(\lambda)},
$$


where

(A.4a)

$$
L_{ \pm}(\lambda)=e^{-H_{ \pm}(\lambda)} \prod_{n=1}^{\infty}\left(\left[1-\left(\frac{k}{n \pi}\right)^{2}\right]^{1 / 2} \mp \frac{i \lambda}{n \pi}\right) e^{ \pm i \lambda / n \pi},
$$

$$
H_{ \pm}(\lambda)=\mp \frac{i \lambda}{\pi}\left[1-\gamma+\ln \frac{2 \pi}{k}\right] \pm \frac{\lambda d}{2}+\frac{\alpha d}{\pi} \cos ^{-1} \frac{ \pm \lambda}{k},
$$

where $\gamma$ is the Euler's constant and

$$
F_{ \pm}(\lambda)=\frac{1}{2 \pi i} \sum_{n=1}^{\infty} \frac{r_{01}^{n}}{n} \int_{-\infty}^{\infty} \frac{\exp \left(-2 n \sqrt{\xi^{2}-k^{2}}\right)}{\xi \pm \lambda} d \lambda .
$$

Defining

$$
\phi_{+}(\lambda)=\frac{1}{2 \pi} \int_{-\infty}^{0} e^{-i \lambda x} d \lambda=-\frac{1}{2 \pi i \lambda}
$$

gives the solution to the dual integral equations as

$$
\sigma(\lambda)=\frac{\varepsilon_{0}+\varepsilon_{1}}{2} \frac{\phi_{+}(\lambda)}{G_{+}(\lambda) G_{-}(0)}
$$

and the Fourier transform of the potential as

$$
\phi(\lambda)=\frac{\phi_{+}(\lambda) G_{-}(\lambda)}{G_{-}(0)} .
$$

Thus, the potential in region 0 or the air region is given by

$$
\Phi_{0}^{(0)}(X, Y)=\int_{-\infty}^{\infty} \phi_{+}(\lambda) \frac{G_{-}(\lambda)}{G_{-}(0)} e^{i \lambda X-\alpha(Y-1)} d \lambda .
$$

The potential in region 1 or the dielectric region can be obtained by matching boundary conditions at $Y=1$ giving

$$
\Phi_{1}^{(0)}(X, Y)=\int_{-\infty}^{\infty} \phi_{+}(\lambda) \frac{G_{-}(\lambda)}{G_{-}(0)}\left[\frac{e^{\alpha(Y-1)}-e^{-2 \alpha-\alpha(Y-1)}}{1-e^{-2 \alpha}}\right] e^{i \lambda X} d \lambda .
$$

The path of integration in (A.8) and (A.9) is defined in the strip of analyticity shared by $\phi_{+}(\lambda)$ and $G_{-}(\lambda)$. The path of integration can be redefined as that shown in Fig. A.1. In the limit when $R \rightarrow 0$, it gives the same integral as above. The advantage of the new definition of integration is that the integrals can be evaluated unambiguously when $k \rightarrow 0$. We bear in mind that the value of $k$ does not enter into the final answer. Thus we can choose it to be any complex number, and in our case, a pure imaginary number as shown in Fig. A.1. When $k=0, G_{ \pm}(\lambda)$ simplifies to

$$
G_{+}(\lambda)=\frac{1}{\Gamma\left(1-\frac{i \lambda}{\pi}\right)} \exp \left\{\frac{i \lambda}{\pi}\left[1+\ln \frac{\pi}{\lambda}\right]-\frac{\lambda}{2}\right.
$$

$$
\left.-\frac{1}{2 \pi i} \sum_{n=1}^{\infty} \frac{r_{01}^{n}}{n}\left[E_{1}(2 n \lambda) e^{2 n \lambda}-E_{1}\left(e^{-i \pi} 2 n \lambda\right) e^{-2 n \lambda}\right]\right\}
$$

and
(A.10b)
$G_{-}(\lambda)=G_{+}\left(\lambda e^{i \pi}\right)$. 


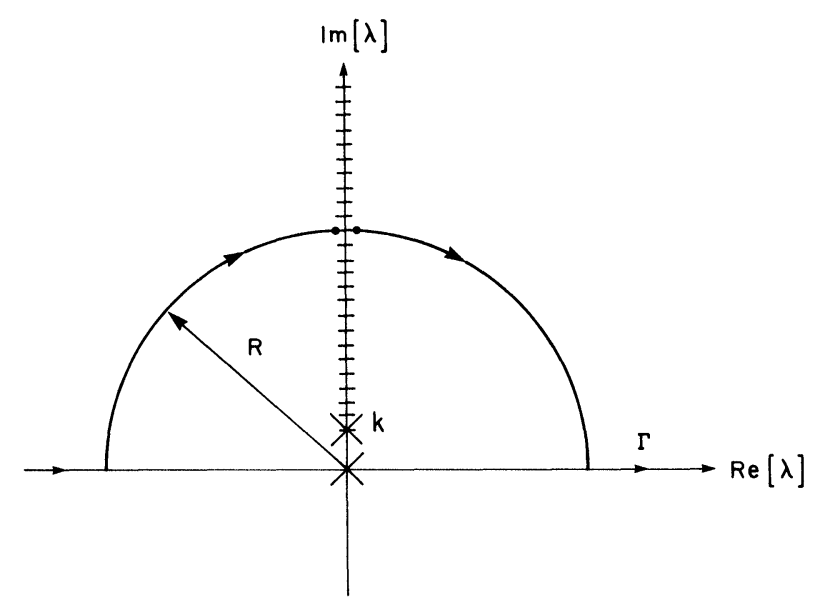

FIG. A.1

When $\left(X^{2}+Y^{2}\right)^{1 / 2} \rightarrow \infty$ in (A.8), most of the contribution to the integral comes from around the origin. $G_{-}(\lambda)$ can be approximated around the origin as

$$
G_{-}(\lambda) \sim G_{-}(0)\left[1+a_{1} \lambda \ln \lambda+a_{2} \lambda+a_{3} \lambda^{2} \ln ^{2} \lambda+a_{4} \lambda^{2} \ln \lambda+O\left(\lambda^{2}\right)\right], \quad \lambda \rightarrow 0,
$$

where

$$
a_{1}=\frac{i}{\pi \varepsilon_{r}}, \quad a_{2}=\frac{i}{\pi \varepsilon_{r}}\left[-A+\gamma+\frac{i \pi}{2}\right]
$$

$$
a_{3}=-\frac{1}{2 \pi^{2} \varepsilon_{r}^{2}}, \quad a_{4}=-\frac{1}{\pi^{2} \varepsilon_{r}^{2}}\left[-A+\gamma+\frac{i \pi}{2}\right],
$$

for which $A$ is defined in (14b). When (A.11a) is substituted into (A.8), and each term evaluated along $\Gamma$, and letting $R \rightarrow 0$ eventually, we obtain the asymptotic expansion of the edge solution as

$$
\Phi_{0}^{(0)}(X, Y) \sim \frac{1}{\pi} \tan ^{-1} \frac{Y}{X}-\frac{1}{\pi^{2} \varepsilon_{r}}\left\{\frac{X}{X^{2}+Y^{2}} \tan ^{-1} \frac{Y}{X}\right.
$$

$$
\begin{array}{r}
\left.-\frac{Y}{X^{2}+Y^{2}}\left[\ln \left(X^{2}+Y^{2}\right)^{1 / 2}+A\right]+\frac{\pi\left(\varepsilon_{r}-1\right) X}{X^{2}+Y^{2}}\right\}, \\
\left(X^{2}+Y^{2}\right)^{1 / 2} \rightarrow \infty .
\end{array}
$$

For the interior expansion of the edge-solution, we notice that the term in the square bracket of (A.9) is an even function of $\alpha$ and thus is branch-point free. When $X \rightarrow-\infty$, the path of integration can be deformed downward picking up the singularity due to $\phi_{+}(\lambda)$ at the origin and the singularities given by $1-e^{-2 \alpha}=0$. Since the latter singularities are never on the real axis, they contribute to exponentially small terms. The contribution due to the pole at the origin is $Y$ and the asymptotic expansion is as shown in (24).

The asymptotic expansion of the integral term in (32a) can be obtained similarly. The path of integration is taken to be along $\Gamma$ with $R \rightarrow 0$ eventually. Thus the integral is given by

$$
\text { (A.13) } \quad I_{1}=\lim _{R \rightarrow 0} \frac{1}{2} \int_{\Gamma} \frac{\phi_{+}(\lambda) G_{-}(\lambda)}{i \lambda G_{-}(0)}\left[e^{-\alpha(Y-1)+i \lambda X}-\varepsilon_{r}(Y-1)-1\right] d \lambda .
$$


The latter two terms in the square bracket evaluate to

(A.14) $-\frac{1}{2} \int_{\Gamma} \frac{\phi_{+}(\lambda) G_{-}(\lambda)}{i \lambda G_{-}(0)}\left[\varepsilon_{r}(Y-1)+1\right] d \lambda \sim-\frac{\left[\varepsilon_{r}(Y-1)+1\right]}{2 \pi \varepsilon_{r}}[\ln R-A+\gamma]$,

$$
R \rightarrow 0 \text {. }
$$

To evaluate the first term in the square bracket when $\left(X^{2}+Y^{2}\right)^{1 / 2} \rightarrow \infty$, we substitute (A.11) into the integral. It can be shown that

$$
\begin{aligned}
\frac{1}{4 \pi} \int_{\Gamma} \frac{1}{\lambda^{2}}\left(1+a_{1} \lambda \ln \lambda+a_{2} \lambda+\cdots\right) e^{i \lambda X-\alpha(Y-1)} d \lambda \\
\sim \frac{Y-1}{2 \pi} \ln \left[R\left(X^{2}+Y^{2}\right)^{1 / 2}\right]+\frac{X}{2 \pi} \tan ^{-1} \frac{Y}{X}+\frac{Y-1}{2 \pi}(\gamma-1) \\
\quad-\frac{1}{2 \pi}+\frac{\ln R}{2 \pi \varepsilon_{r}}-\frac{1}{2 \pi^{2} \varepsilon_{r}} \tan ^{-1} \frac{Y}{X}\left[\ln \left(X^{2}+Y^{2}\right)^{1 / 2}+A\right] \\
+\frac{1}{2 \pi \varepsilon_{r}}\left[\ln \left(X^{2}+Y^{2}\right)^{1 / 2}+\gamma\right], \quad R \rightarrow 0 .
\end{aligned}
$$

Hence,

$$
I_{1} \sim \frac{X}{2 \pi} \tan ^{-1} \frac{Y}{X}-\frac{Y}{2 \pi}-\frac{1}{2 \pi \varepsilon_{r}}\left[\frac{1}{\pi} \tan ^{-1} \frac{Y}{X}-1-\varepsilon_{r}(Y-1)\right]\left[\ln \left(X^{2}+Y^{2}\right)^{1 / 2}+A\right],
$$

$$
\left(X^{2}+Y^{2}\right)^{1 / 2} \rightarrow \infty
$$

Equation (A.16) together with (A.12) enables us to find the asymptotic approximation of $\Phi_{0}^{(2)}(X, Y)$ given by

$$
\begin{aligned}
& \Phi_{0}^{(2)}(X, Y) \sim \frac{1}{2 \pi} \ln \left[\frac{\left(X^{2}+Y^{2}\right)^{1 / 2}}{8}\right]\left[Y-\frac{1}{\pi \varepsilon_{r}} \tan ^{-1} \frac{Y}{X}-1+\frac{1}{\varepsilon_{r}}\right] \\
& -\frac{Y}{2 \pi^{2} \varepsilon_{r}\left(X^{2}+Y^{2}\right)}\left\{X\left[\ln \left(X^{2}+Y^{2}\right)^{1 / 2}+A\right]+Y \tan ^{-1} \frac{Y}{X}+\pi\left(\varepsilon_{r}-1\right) Y\right\},
\end{aligned}
$$

which is seen to satisfy the matching condition supplied by (25). The interior expansion of $\Phi_{1}^{(2)}(X, Y)$ can be found similarly and is exponentially small.

The charge stored in the edge region in a strip of width $W$ is given by

$$
I_{2}=\int_{-W}^{0} \Sigma^{(0)}(X) d X
$$

From (A.1b) and (A.6), we derive that

$$
\begin{aligned}
I_{2} & =\frac{\varepsilon_{0}+\varepsilon_{1}}{2} \int_{-\infty}^{\infty} \frac{\phi_{+}(\lambda)}{i \lambda G_{+}(\lambda) G_{-}(0)}\left[1-e^{-i \lambda W}\right] d \lambda \\
& =-\frac{\varepsilon_{0}+\varepsilon_{1}}{2} \int_{-\infty}^{\infty} \frac{\phi_{+}(\lambda)}{i \lambda G_{+}(\lambda) G_{-}(0)} e^{-i \lambda W} d \lambda .
\end{aligned}
$$

The latter equality follows from that the first term, which is analytic in the upper half-plane where the path of integration is defined, evaluates to zero. When $W \rightarrow \infty$, we 
approximate $G_{+}^{-1}(\lambda)$ around the origin as

(A.20a) $G_{+}^{-1}(\lambda) \sim G_{+}^{-1}(0)\left[1+b_{1} \lambda \ln \lambda+b_{2} \lambda+b_{3} \lambda^{2} \ln ^{2} \lambda+b_{4} \lambda^{2} \ln \lambda+\cdots\right], \quad \lambda \rightarrow 0$, where

$$
b_{1}=\frac{i}{\pi \varepsilon_{r}}, \quad b_{2}=\frac{i}{\pi \varepsilon_{r}}\left[-A+\gamma-i \frac{\pi}{2}\right]
$$

$$
b_{3}=-\frac{1}{2 \pi^{2} \varepsilon_{r}^{2}}, \quad b_{4}=-\frac{1}{\pi^{2} \varepsilon_{r}^{2}}\left[-A+\gamma-i \frac{\pi}{2}\right] \text {. }
$$

Consequently, we have the asymptotic expansion of (A.19) as

$$
I_{2} \sim-\varepsilon_{1}\left\{W+\frac{1}{\pi \varepsilon_{r}} \ln W+\frac{A}{\pi \varepsilon_{r}}+\frac{1}{\pi^{2} \varepsilon_{r}^{2} W}[\ln W+A]+\cdots\right\},
$$

which when substituted into (36) gives rise to its approximation.

Appendix B. We want to find the edge expansion of

$$
V_{0}(\rho, z) \sim V_{0}^{(0)}(\rho, z)+\delta \ln \delta V_{0}^{(1)}(\rho, z)+\delta V_{0}^{(2)}(\rho, z), \quad \delta \rightarrow 0
$$

The edge expansion of $V_{0}^{(0)}(\rho, z)$ and $V_{0}^{(1)}(\rho, z)$, when written in terms of the Hankel transform as in (8) and (21), can be obtained easily using the approximation of Eason and associates [16]. The edge expansion of $V_{0}^{(2)}(\rho, z)$ involves more intricate manipulation. Using Eason's approximation and the elliptic integral approximation [18], we can rewrite the first two terms of (23) as

$$
\begin{aligned}
\frac{2}{\pi^{2} \varepsilon_{r}} & \int_{0}^{1}\left\{\frac{z}{\left(1-b^{2}\right)\left[(\rho-b)^{2}+z^{2}\right]}-\frac{z}{2(1-b)\left[(\rho-1)^{2}+z^{2}\right]}\right\} d b \\
& -\frac{1}{2 \pi^{2} \varepsilon_{r}} \int_{0}^{1}\left[\ln \left(\frac{1-b^{2}}{16}\right)+1\right] \frac{z}{\left[(\rho-b)^{2}+z^{2}\right]} d b \\
& -\frac{1}{\pi^{2} \varepsilon_{r}} \int_{0}^{1}\left\{\frac{z(\rho-b)}{b\left[(\rho-b)^{2}+z^{2}\right]\left(1-b^{2}\right)}-\frac{z(\rho-1)}{2\left[(\rho-1)^{2}+z^{2}\right](1-b)}\right\} d b .
\end{aligned}
$$

The first term in the above equation evaluates, in terms of edge variables, to

$$
-\frac{X \tan ^{-1}(Y / X)-Y \ln \left[\delta\left(X^{2}+Y^{2}\right)^{1 / 2}\right]}{\pi^{2} \varepsilon_{r} \delta\left(X^{2}+Y^{2}\right)}+\frac{1}{2 \pi^{2} \varepsilon_{r}} \tan ^{-1} \frac{Y}{X}
$$

The second term, with the help of the dilogarithm function [19], evaluates to

$$
-\frac{1}{2 \pi^{2} \varepsilon_{r}} \tan ^{-1} \frac{Y}{X} \ln \left[\delta\left(X^{2}+Y^{2}\right)^{1 / 2}\right]-\frac{1}{2 \pi^{2} \varepsilon_{r}}\left(\ln \frac{1}{8}+1\right) \tan ^{-1} \frac{Y}{X}
$$

The third term evaluates to

$$
-\frac{1}{2 \pi^{2} \varepsilon_{r}} \frac{X Y \ln \left[\delta\left(X^{2}+Y^{2}\right)^{1 / 2}\right]}{\left(X^{2}+Y^{2}\right)}-\frac{1}{2 \pi^{2} \varepsilon_{r}} \frac{Y^{2} \tan ^{-1}(Y / X)}{\left(X^{2}+Y^{2}\right)} .
$$


It can be shown that the errors in the above are of order less than 1 . As a result, the edge expansion of $V_{0}^{(2)}(\rho, z)$ is

$$
V_{0}^{(2)}(1+\delta X, \delta Y)
$$

$$
\sim \frac{1}{\delta \pi^{2} \varepsilon_{r}\left(X^{2}+Y^{2}\right)}\left\{Y\left(\ln \left[\delta\left(X^{2}+Y^{2}\right)^{1 / 2}\right]+A\right)-X \tan ^{-1} \frac{Y}{X}-\pi\left(\varepsilon_{r}-1\right) X\right\}
$$

$$
\begin{aligned}
& -\frac{1}{2} \ln \left[\frac{\delta\left(X^{2}+Y^{2}\right)^{1 / 2}}{8}\right]\left[\varepsilon_{r}-1+\frac{1}{\pi} \tan ^{-1} \frac{Y}{X}\right] / \pi \varepsilon_{r} \\
& -\frac{Y}{2\left(X^{2}+Y^{2}\right)}\left[Y \tan ^{-1} \frac{Y}{X}+X \ln \left[\delta\left(X^{2}+Y^{2}\right)^{1 / 2}\right]+\pi\left(\varepsilon_{r}-1\right) Y+A X\right] / \pi^{2} \varepsilon_{r}
\end{aligned}
$$

The above expression, together with the edge expansion of $V_{0}^{(0)}$ and $V_{0}^{(1)}$, gives rise to the expression in (25).

The edge-expansion of (33) and (34) can be similarly obtained. The edgeexpansion of $q_{0}^{(0)}$ and $q_{0}^{(1)}$ can be easily derived. For $q_{0}^{(2)}$, we again rewrite the first term of it before taking the $z \rightarrow 0$ limit as

$$
\begin{gathered}
I=\frac{8 \varepsilon_{0}}{\pi \varepsilon_{r}} \int_{0}^{1} d b\left\{\frac{b E(b)}{1-b^{2} \rho} \int_{0}^{\infty} \lambda J_{0}(\lambda b) J_{1}(\lambda \rho) e^{-\lambda z} d \lambda\right. \\
-\frac{1}{2(1-b)} \rho \int_{0}^{\infty} \lambda J_{0}(\lambda) J_{1}(\lambda \rho) e^{-\lambda z} d \lambda \\
-\frac{1}{4}\left[\frac{\rho}{1-b}\left(\frac{\rho-b}{(\rho-b)^{2}+z^{2}}-\frac{\rho-1}{(\rho-1)^{2}+z^{2}}\right)\right. \\
\quad+\frac{1}{2} \ln 8 \frac{\rho-b}{(\rho-b)^{2}+z^{2}}-\frac{1}{2} \ln (1-b) \frac{\rho-b}{(\rho-b)^{2}+z^{2}} \\
\left.+\frac{2 \varepsilon_{0}}{\pi \varepsilon_{r}} \int_{0}^{1} \begin{array}{c}
\left.d b\left\{\frac{\rho}{1-b}\left(\frac{\rho-b}{(\rho-b)^{2}+z^{2}}-\frac{\rho-1}{(\rho-1)^{2}+z^{2}}\right)+\frac{1}{2} \ln \left(\frac{(\rho-b)^{2}+z^{2}}{(\rho-1)^{2}+z^{2}}\right)\right]\right\} \\
\quad-\frac{1}{2} \ln (1-b) \frac{\rho-b}{(\rho-b)^{2}+z^{2}} \\
+z^{2}-\frac{1}{2(1-b)} \ln \left(\frac{(\rho-b)^{2}+z^{2}}{(\rho-1)^{2}+z^{2}}\right)^{1 / 2}
\end{array}\right\} .
\end{gathered}
$$

Using the approximation of Eason et al. [16] for the Lipschitz-Hankel integrals, and the elliptic integral approximation, it can be shown that when $(1-\rho)$ and $z \rightarrow 0$, the first integral in the above tends to a constant given by

$$
\frac{2 \varepsilon_{0}}{\pi \varepsilon_{r}} \int_{0}^{1}\left[4 b\left(\frac{E(b)}{1-b^{2}}\right)^{2}-\frac{1}{(1-b)^{2}}+\frac{1}{1-b}\left(\ln \frac{1-b}{8}+1\right)\right] d b
$$

which is shown by Wigglesworth [20] to be

$$
\frac{\varepsilon_{0}}{\pi \varepsilon_{r}}\left(\ln \frac{1}{8}+1\right)^{2}
$$


For the latter integral in (B.4), the first term can be integrated in terms of edge variables giving

$$
\int_{0}^{1} d b \frac{\rho}{1-b}\left(\frac{\rho-b}{(\rho-b)^{2}+z^{2}}-\frac{\rho-1}{(\rho-1)^{2}+z^{2}}\right)
$$

$$
\sim\left(\frac{Y \tan ^{-1}(Y / X)+X \ln \left[\delta\left(X^{2}+Y^{2}\right)^{1 / 2}\right]}{\left[X^{2}+Y^{2}\right] \delta}(1+\delta X)-1\right), \quad \delta \rightarrow 0 .
$$

The latter terms can be integrated similar to (B.2) giving

$$
\begin{gathered}
\frac{\ln 8}{2} \int_{0}^{1} d b \frac{\rho-b}{(\rho-b)^{2}+z^{2}} \sim-\frac{\ln 8}{2} \ln \left[\delta\left(X^{2}+Y^{2}\right)^{1 / 2}\right], \quad \delta \rightarrow 0, \\
-\frac{1}{2} \int_{0}^{1} d b \ln (1-b) \frac{\rho-b}{(\rho-b)^{2}+z^{2}} \sim \frac{\ln ^{2}\left[\delta\left(X^{2}+Y^{2}\right)^{1 / 2}\right]}{4} \\
-\frac{\left[\tan ^{-1}(Y / X)\right]^{2}}{4}+\frac{\pi^{2}}{12}, \quad \delta \rightarrow 0 . \\
-\frac{1}{2} \int_{0}^{1} d b \frac{1}{1-b} \ln \left(\frac{(\rho-b)^{2}+z^{2}}{(\rho-1)^{2}+z^{2}}\right)^{1 / 2} \\
\sim-\frac{\ln ^{2}\left[\delta\left(X^{2}+Y^{2}\right)^{1 / 2}\right.}{4}+\frac{\left[\tan ^{-1}(Y / X)\right]^{2}}{4}-\frac{\pi^{2}}{12}, \quad \delta \rightarrow 0 .
\end{gathered}
$$

As a result, when $X=-W$

$$
\lim _{Y \rightarrow 0} I \sim-\frac{2 \varepsilon_{0} \ln (\delta W)}{\pi \varepsilon_{r} \delta W}-\frac{\varepsilon_{0}}{\pi \varepsilon_{r}}(\ln 8-2) \ln \delta W-\frac{2 \varepsilon_{0}}{\pi \varepsilon_{r}}+\frac{\varepsilon_{0}}{\pi \varepsilon_{r}}\left(\ln \frac{1}{8}+1\right)^{2} .
$$

The above, together with the edge expansions of the Lipschitz-Hankel integrals give rise to the expression in (39).

\section{REFERENCES}

[1] G. KirchHoff, Monatsb. Deutsch Akad. Wiss, Berlin, 1877, pp. 144-162.

[2] W. IgnAtowsky, Akad. Nauk SSSR, Mat. Inst. Trudy, 2, 3, 1932, pp. 1-104.

[3] G. Polya AND G. Szegö, Isopermetric Inequalities in Mathematical Physics, Princeton University Press, Princeton, NJ, 1951.

[4] Y. Nomura, The electrostatic problems of two equal parallel circular plates, Proc. Phys. Math. Soc. Japan, 3, 23 (1941), pp. 168-180.

[5] J. C. Cooke, The coaxial circular disc problem, Z. Angew. Math. Mech., 38 (1958), pp. 349-356.

[6] V. Hutson, The circular plate condenser at small separations, Proc. Cambridge Philos. Soc., 59 (1963), pp. 211-224.

[7] F. LEPPINGTON AND H. LEVINE, On the capacity of the circular disk condenser at small separation, Proc. Cambridge Philos. Soc., 68 (1970), pp. 235-254.

[8] S. SHAw, Circular-disk viscometer and related electrostatic problems, Phys. Fluids, 13 (1970), pp. 1935-1947.

[9] T. ITOH AND R. MitTRA, A new method for calculating the capacitance of microwave integrated circuits, IEEE Trans. Micro. Theory Tech., MTT-21 (1973), pp. 431-432.

[10] W. C. CHEW AND J. A. KONG, Effects of fringing fields on the capacitance of circular microstrip disk, IEEE Trans. Micro. Theory Tech., MTT-28 (1980), pp. 98-104.

[11] - Asymptotic formula for the capacitance of two parallel plates separated by a dielectric slab, Math. Proc. Camb. Philos. Soc., 89 (1981), pp. 373-384. 
[12] M. VAN Dyke, Perturbation Methods in Fluid Mechanics, Parabolic Press, Stanford, CA, 1975.

[13] J. C. Cooke, Circular disc between two parallel planes close together, Proc. Royal Soc. Edin., 73A, 9 (1974/75), pp. 149-160.

[14] S. SHAw, Circular disc viscometer and related electrostatic problems, Doctoral thesis, Stanford University, Stanford, CA, 1969.

[15] MACSYMA Reference Manual, Laboratory for Computer Science, Massachusetts Institute of Technology, Cambridge, MA, 1977.

[16] G. EASON, B. Noble AND I. N. SNeddon, On certain integrals of Lipschitz-Hankel type involving products of Bessel functions, Philos. Trans. Royal Soc. London, A (1955), pp. 529-551.

[17] B. NoBle, The Wiener-Hopf Technique, Pergamon Press, London, 1958.

[18] I. S. Gradshteyn AND I. W. Ryzhik, Tables of Integrals, Series and Products, Academic Press, New York, 1965.

[19] L. LEWIN, Dilogarithms and Associated Functions, MacDonald, London, 1958.

[20] L. A. Wigglesworth, Comments on "circular viscometer and related electrostatic problems", Phys. Fluids, 15 (1972), pp. 718-719. 
Reproduced with permission of the copyright owner. Further reproduction prohibited without permission. 